Original Research

\title{
Reevaluating the Sperm Nuclear Chromatin Decondensation Test by Sodium Thioglycolate of Stallions Spermatozoa
}

\author{
Ricardo Hartley ${ }^{\mathrm{a}, \mathrm{b}}$, Pablo Aros ${ }^{\mathrm{b}}$, Eduardo Bustos-Obregón ${ }^{\mathrm{c}}$, Fernando Romero ${ }^{\mathrm{d}}$, \\ Marco Alvarenga ${ }^{e}$, Alfredo Ramírez-Reveco ${ }^{\mathrm{b}, *}$ \\ a Programa Doctorado en Ciencias Mención Biología Celular y Molecular Aplicada, Universidad de la Frontera, Temuco, Chile \\ ${ }^{\mathrm{b}}$ Laboratorio de Criobiología y Análisis de Funcionalidad Espermática, Instituto de Ciencia Animal, Facultad de Ciencias Veterinarias, Universidad Austral de Chile, \\ Campus Isla Teja, Valdivia, Chile \\ ${ }^{\mathrm{c}}$ Laboratorio Biología de la Reproducción y del Desarrollo, Facultad de Medicina, Universidad de Chile, Santiago, Chile \\ 'Departamento de Ciencias Preclínicas, Facultad de Medicina, Universidad de la Frontera, Temuco, Chile \\ e Faculdade de Medicina Veterinária e Zootecnia, Sao Paulo State University-UNESP, Botucatu, Sao Paulo, Brazil
}

\section{A R T I C L E I N F O}

\section{Article history:}

Received 8 June 2015

Received in revised form

18 September 2015

Accepted 12 October 2015

Available online 23 October 2015

\section{Keywords:}

Stallion

Sperm nucleus

Chromatin compaction

Sodium thioglycolate

\begin{abstract}
A B S T R A C T
The nucleus of mammalian spermatozoa is mainly composed of chromatin associated with protamines: highly basic proteins (around $7 \mathrm{kDa}$ ). These highly basic proteins, due to their cysteine content, can participate in the generation of disulfide bond. These characteristics permit typical condensed nuclear structure in mature spermatozoa, where the DNA becomes organized in compacted units, similar to nucleosomes, but with $60 \mathrm{~kb}$ of DNA. This shape is ultimately assumed in the epididymal maturation, and the level of compaction is closely related to epididymal function. In the present work, we present a modified method to evaluate sperm decompaction using sodium thioglycolate (ST). Stallion sperm were exposed to different ST concentrations and were embedded in agarose as a supportive medium. With the use of agarose, it was easier to identify patterns of decompaction with ST, and, thus, the use of a permeabilizing solution was not necessary. This was due to the utilization of ST to evaluate chromatin compaction of stallion sperm physically permeabilized and embedded in agarose matrix.
\end{abstract}

(c) 2015 Elsevier Inc. All rights reserved.

\section{Introduction}

In the seminiferous tubule of testis, where spermiogenesis occurs, spermatids nuclei undergo complex morphologic, biochemical, and physiological changes to complete their development. One of these changes is related to nuclei condensation due to the exchange of histones primarily by transition proteins, after highly basic arginine and cysteine rich protamines. Later, the nucleus will be composed of highly compacted chromatin during the epididymal transit because of the formation of

\footnotetext{
* Corresponding author at: Alfredo Ramírez-Reveco, Instituto de Ciencia Animal, Facultad de Ciencias Veterinarias, Universidad Austral de Chile, Campus Isla Teja, Valdivia, Chile.

E-mail address: alfredoramirez@uach.cl (A. Ramírez-Reveco).
}

disulphide bonds between free sulfhydryls on cysteine of protamines that will be oxidized to form disulphide bonds [1]. This produces protamine-DNA chromatin, generating tightly packed toroidal-like structures, containing up to 60 $\mathrm{kb}$ of DNA, in which the transcription and repair activities are inactivated and also related to morphology of sperm head and maturation state of sperm during epididymal transit [2].

As one of the features that can provide functional information of the sperm, several approaches for assessing the presence of disulfide bonds have been tested involving reductant agents such as including dithiothreitol (DTT) + sodium dodecyl sulfate (SDS) detergent, and sodium thioglycolate (ST) among others [3,4]; being their effect in the sperm nucleus mainly evaluated as changes in sperm head size. 
Sodium thioglycolate was chosen because, as was published by Fornés and Bustos-Obregón [5], the fact that it could freely pass the plasma membrane. In stallion sperm, ST tests have been used to study compaction level [6]. However, their observation and evaluation is not easy, because mainly to a weak and nonspecific staining, heterogeneous response of sperm to decondensation process, that evidenced it with several patterns of staining (color and size of halo). Moreover, to date, there is no evidence that the sodium thioglycolate across freely and without resistance through the plasma membrane of the sperm. If not the case, part of then response to decondensation depends on if the sodium thioglycolate effectively was incorporated to citosol and later reacting with sperm chromatin, aspect that was evaluated in this work.

Here, we propose the use of a new protocol of ST as a reducing agent for disulphide bonds, overcoming the lack of the effect in sperm with plasma membrane integrity, whereas with the original protocol, only sperm with their membrane disrupted presented reactive characteristics. This differs from the work of Fornés and Bustos-Obregón [5], in which all cells are expected to react with ST, and owing to the fact that the conditions in which the sperm cells are immobilized and attached to the slide for observation are different, being the agarose a supportive medium for controlled and homogeneous sperm sample decompaction.

\section{Materials and Methods}

\subsection{Sample Preparation and Selection}

In this study, only frozen semen samples were used. The samples were obtained from ejaculates of five stallions, with fertility probed with frozen semen, and with ages between 7 and 16 years old, from Heavy Draft breeds, property of Haras Militar Pupunahue (a member of the Fondef D08I1076 project), which explicitly approved the use of the samples for scientific research purposes.

Qualified samples were cryopreserved according to standard procedure, using a BotuCrio freezing extender. The ejaculates were collected using an artificial vagina, filtered to remove gel, diluted 1:1 in prewarmed skimmed milk (isothermal condition), and centrifuged at $1,000 \mathrm{~g}$ for 20 minutes. Postcentrifugation sperm pellets were suspended in extenders previously tempered at $20^{\circ} \mathrm{C}$, packed at $50 \times 10^{6} \mathrm{sperm} / \mathrm{mL}$ in straws of $0.5 \mathrm{~mL}$, and cooled to $5^{\circ} \mathrm{C}$ for 90 minutes. They were subsequently exposed to liquid nitrogen vapors for 20 minutes at $4 \mathrm{~cm}$ over the liquid nitrogen level and finally submerged and stored in liquid nitrogen until analysis.

The cryopreservation samples were thawed at $37^{\circ} \mathrm{C}$ for 30 seconds before their evaluation.

In this study, only cryopreserved samples with a postthawing sperm vitality evaluation over $40 \%$ were considered.

\subsection{Sperm Vitality Evaluation}

Vitality of the fresh sperm was evaluated using an eosin/ nigrosin stain. A drop of the semen sample was mixed with a larger drop of eosin $Y(2.5 \%)$, and nigrosin (5\%) stains on a prewarmed slide with an applicator stick and a thin smear was made with another slide. After air-drying, the smear was observed $(\times 40)$ with an LED illuminated view microscope (Nikon E200) for unstained heads of the spermatozoa (live) and stained or partially stained heads of the spermatozoa (nonviable). A total of 200 spermatozoa were counted to determine the percentage of live and nonviable spermatozoa. The average of three observations was considered a single data point.

In the laboratory, both cooled and thawed sperm samples were assessed using acridine orange (AO)/propidium iodide (PI) double staining. Sperm samples were mixed $(1: 1)$ on a tempered microscope slide with a staining solution $\mathrm{AO} / \mathrm{PI}$ (20 and $10 \mu \mathrm{M}$, respectively) and were immediately analyzed using the viability module from the CASA System (Sperm Class Analyzer; Microptic, Spain) and observed at $\times 10$ with the aforementioned LED illuminated view microscope (Nikon E200). During this procedure, viable and dead spermatozoa were observed with differential fluorescent emission in the head spermatozoa (green [525 nm] for viable and red [620 nm] for nonviable spermatozoa). Viability percentages were established from a count of at least 500 spermatozoa in each sample.

\subsection{Sodium Thioglycolate Treatment}

\subsubsection{Fornés and Bustos-Obregón (protocol A)}

A volume of $1 \mathrm{~mL}$ of $0.4-\mathrm{M}$ ST pH 9 was added to $1 \mathrm{~mL}$ of $1 \times 10^{4}$ sperm suspension and incubated at room temperature for 15 minutes. The reaction was stopped using a 10 -fold dilution with cold phosphate buffer $(0.05 \mathrm{M}, \mathrm{pH}$ 7.2), centrifuged at $760 \mathrm{~g}$ for 5 minutes, and the pellet was then resuspended in phosphate-buffered saline [5].

\subsubsection{Modified Protocol (B)}

A number of $1 \times 10^{4}$ sperm in $80 \mu \mathrm{L}$ of low melting point (LMP) agarose $1 \%$ was mixed at $37^{\circ} \mathrm{C}$ (volume of $2-\mu \mathrm{L}$ spermatozoa in $80-\mu \mathrm{L}$ agarose) and placed in $40 \mu \mathrm{L}$ over a $1 \%$ agarose LE glass slide prewarmed at $37^{\circ} \mathrm{C}$, covered with coverslip, and left to cool for a maximum of 5 minutes at $4^{\circ} \mathrm{C}$. The plasma membrane integrity was evaluated in parallel set of slides by AO/PI double staining in epifluorescent microscope.

In the not stained (AO/PI) set of slides, a drop of ST was added at different concentrations and incubated for 15 minutes (for the dose response experiment at a concentration of $0.2 \mathrm{mM}$ until $200 \mathrm{mM}$ ) or 30 minutes (for standardized protocol, concentration $0.5 \mathrm{M}$ ). After incubation, the slide was dehydrated in ethanol $(95 \%$ and 100\%, 5-minutes each), air dried, and stained with Wright eosin methylene blue stain (Merck). The samples were stained until a layer of oxidized stain was observed (between 10 and 30 minutes), then washed with tap water, air dried, and mounted on a hydrophobic mountain medium. Resume of protocols are shown in Supplementary Fig. 1. For control of reductant agent, sperm cells were incubated in DTT, 2-Mercaptoethanol (BME), and ST at 0.5, 0.05, and $0.005 \mathrm{M}$ diluted in distilled water, following the protocol just indicated. This is showed in Supplementary Fig. 2. 
A

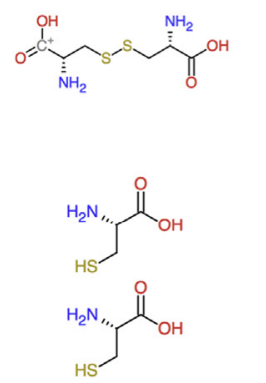

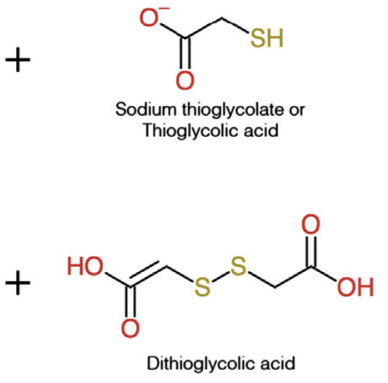

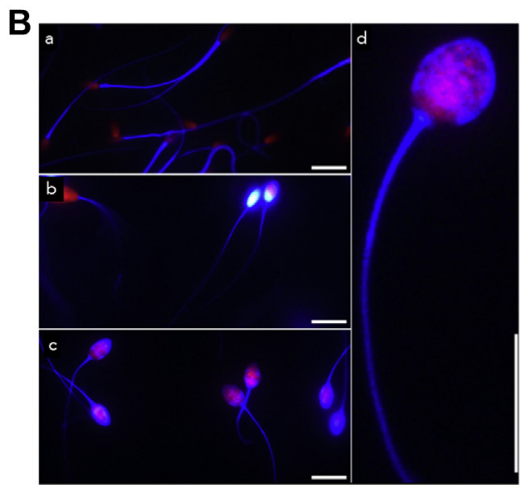

Fig. 1. (A) shows the reaction scheme of a disulfide bond between cysteine when sodium thioglycolate is added (draw with ChemDoodle Web Components [online]. http://web.chemdoodle. com). The two free $\mathrm{SH}$ groups produced can react with reactives such as monobromobimane (mBBr) or bibromobimane (bBBr). (B) shows the SH groups created after incubation with ST being visualized with bBBr and propidium iodine staining: Control sample, without preincubation with ST (a), preincubated with ST sample (b and c), and higher magnification in (d). ST, sodium thioglycolate.

\subsubsection{SH Group Staining by Bibromobimane}

For bibromobimane (bBBr) staining, the protocol B was used with modifications, specifically, Wright staining was replaced with 2-mM bBBr (diluted in distilled water) after incubation with $10-\mu \mathrm{M}$ PI.

\subsection{Nuclear Observation}

Wright eosin methylene blue staining was chosen because of its polychromatic content, where eosin stains basic cell component (e.g., protamines) and methylene blue mainly stains acid cell components (e.g., DNA). For computational analysis of protocol $A$, the viability module from the CASA System was used (Sperm Class Analyzer; Microptic). The size area detection (halo) was calibrated depending on signal brightness; this is due the CASA system is able to identify ranges of sizes. For our study, the range considered for decompacted sperm was $20 \mu \mathrm{m}^{2}$. In addition, for manual user count, a range from strong bluestained nuclei to swelled nuclei was considered as reacted. A pale or celestial blue nuclear stain was considered as not reacted (Supplementary Fig. 2).

\section{Results}

The way a disulfide bond is broken and reacts with ST is shown in Fig. 1A. To check if the disulfide bond was directly affected after the ST treatment, the nuclei were observed using bBBr, as seen in Fig. $1 \mathrm{~B}$, in blue. The increase in the staining of $\mathrm{bBBr}$ and the head size after the treatment mainly is observed in Fig. 1B.

After observing decompaction following the application of protocol A, it was discovered that not all cells reacted. As a result of this finding, viability was tested
A

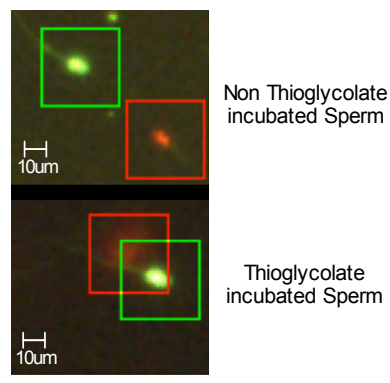

B

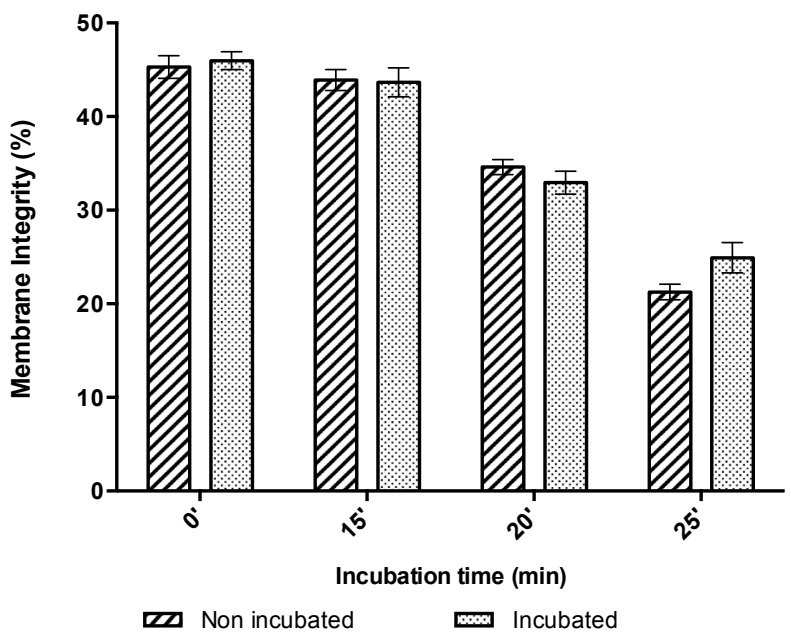

Fig. 2. (A) Sodium thioglycolate sperm uptake. Left, microphotography shows that only red sperm (without membrane integrity) react with sodium thioglycolate. (B) Graph shows that membrane integrity is not influenced by sodium thioglycolate incubation, and only membrane disrupted sperm suffer decondensation. Mann-Whitney test statistical analysis, $P \leq .05$. Plotted mean with SEM. SEM, standard error of the mean. 

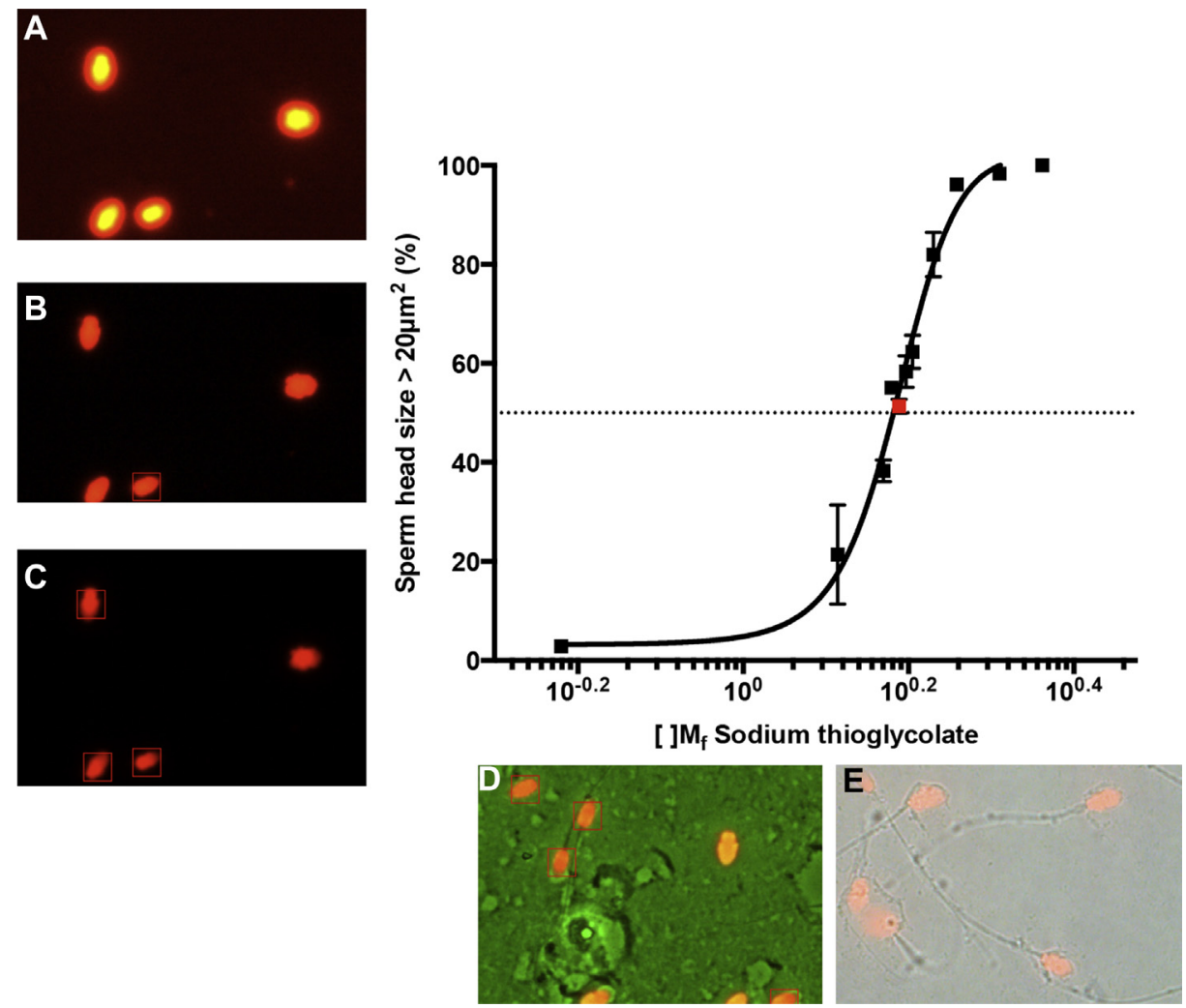

Fig. 3. Graph: Dose response of sperm head to different treatment of sodium thioglycolate. Head increase size was evaluated with Sperm Class Analyzer software, vitality module. The compacted core was defined with an area $<20 \mu \mathrm{m}^{2}$. (A-C) describe different configurations of the saturation field selected for analysis, pointing to a type 2 error in (A), (B) proper saturation, and a type 1 error in (C) by insufficient saturation of the image. In (D) and (E), microphotographs of stained smears of different fields with propidium iodide, combining phase contrast, and fluorescence to verify selective nuclear stain. $\mathrm{n}=5$.

after an ST treatment to determine if all the sperm in the solution had reacted. It was observed that only membrane disrupted sperm were decompacted (Fig. 2A) and that incubation time with ST did not influence the plasma membrane disruption (Fig. 2B). Not all cells reacted with ST, and reacted cells often exhibited compromised membrane integrity. This finding suggests the development of a new protocol for pretreating cells and achieving disruption. One protocol tested was to thaw and freeze the sperm sample to disrupt the plasma membrane. Doing this twice produced cryopreserved samples with disrupted membranes, which were later incubated with the protocol $\mathrm{A}$ at different concentrations of ST. Fig. 3 shows that the decompaction level depends on the concentration of ST in sperm with disrupted plasma membranes, with the advantage that with these treatments, no detergent is used to produce decompaction as with SDS + DTT [7].
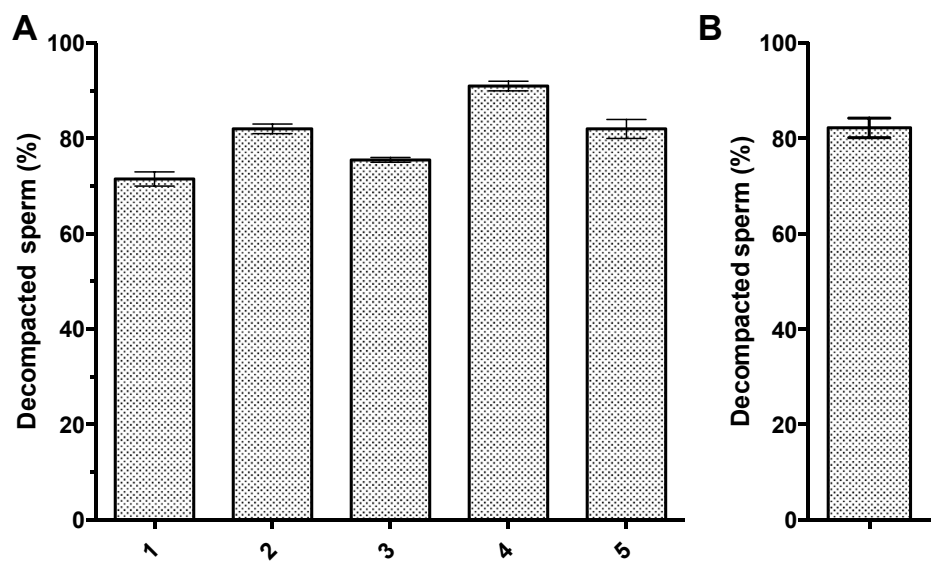

C

Not Decompacted

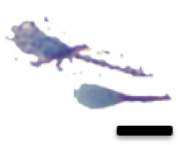

Decompacted

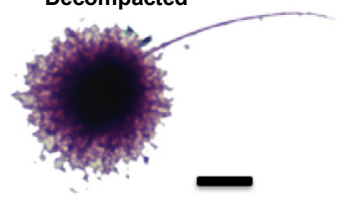

Decompacted

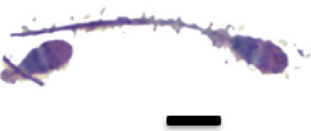

Decompacted

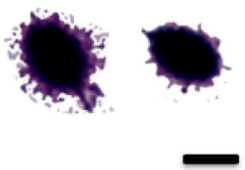

Fig. 4. (A) Comparison of reactivity to sodium thioglycolate (\%) in stallion sperm samples $(n=5)$. (B) Mean of samples. (C) Profiles of reaction classification. Mann-Whitney test statistical analysis, $P \leq .05$. Plotted mean with SEM. SEM, standard error of the mean. 
Moreover, it is recommended the use of ST prepared in distilled water at $\mathrm{pH}$ 6.2, where its redox potential is higher than DTT and 2-Mercaptoethanol (BME) $(-281.7 \mathrm{~V}$ at $\mathrm{pH}$ 6.9 for $\mathrm{ST},-0.33 \mathrm{~V}$ at $\mathrm{pH} 7$ for DTT, and $-0.26 \mathrm{~V}$ at $\mathrm{pH} 7$ for $\mathrm{BME}$ ). With the disruptor pretreatment of the plasma membrane (cycles of freeze and thaw), it is not necessary to use of SDS or other detergents to facilitate the entry and action of reductant agent (Supplementary Fig. 2).

Although it was possible to count decompacted sperm cells after incubation, they tended to group with the reacted sperm on the edges of the slide and nonreacted in the center. This made counting reproducibility difficult. Thus, before treatment, the sperm cells should be attached to an agarose matrix, and later both nuclear proteins and DNA are to be treated and stained. For this purpose, protocol B is suggested, ensuring that all sperm have their membrane disrupted after the cooling process (following the addition of agarose mixed with sperm samples), and before ST treatment. With this protocol, it was possible to count the number of sperm reacted in a reproducible manner by a single individual using the CASA System. Using frozen semen samples from five stallions, the percentage reacted ranged from $77 \%$ to $97 \%$ (Fig. $4 \mathrm{~A}$ ), with an average of $81 \%$ (Fig. 4B).

Finally, considering that the reacted sperms have a head size upper to $20 \mu \mathrm{m}^{2}$, several patterns of descompactation staining were observed (Fig. 4C).

\section{Discussion}

In the mammal sperm nucleus, protamines are the main protein component of chromatin. However, protamines are not equally expressed between mammals [8]. In stallions, protamine one and protamine two variants have been described [9]. However, unlike for humans, where protamine one and two are present in 1:1 ratio, in stallions, the protamine two variant corresponds to $15 \%$ of nuclear protamines [10]. The different content of protamines influences their reactivity to reducing agents, which makes testing different reactants and protocols between species necessary.

No studies related to the concentration of thiol groups have been conducted for stallion sperm. However, in human sperm, protamine P1 was found to contain $81.3 \pm$ $13 \mathrm{mmol}$ thiols $(\mathrm{SH}) / \mathrm{mol}$ and protamine $268.1 \pm 12.3 \mathrm{mmol}$ $(\mathrm{SH}) / \mathrm{mol}[11]$. Such thiol groups are readily oxidized in the air by disulfide. To maintain these groups in the reduced state, another thiol, such as cysteine, glutathione, mercaptoethanol, 2.3-dimercaptopropanol, or thioglycolate, is often added [12].

Diverse tests have been suggested to evaluate the chromatin packaging state in the sperm cell. They are all related to the disulfide bond necessary for their packing. In our case, the striking color shift of human sperm nuclei following the ST test and the observation of "halo" in treated sperm is an expected consequence of the greater availability of anionic sites [4] and the spread of basic proteins from the nuclear core; allowing us to identify compaction status of sperm cryopreserved. Moreover, the use of bBBr permitted us to identify the generation of $-\mathrm{SH}$ groups induced by ST pretreatment, which are related to changes in sperm head size.
In the present work, a new approximation for sperm nuclear compaction state is presented. This could be useful in the evaluation of stallion for which previous parameters did not explain their subfertile status [13].

\section{Conclusions}

Reevaluating the chromatin compaction status with more sensitive and reproducible techniques could deliver new insights related to nuclear integrity and their relationship with sperm fertility potential. Additionally, advancements could be made relating to changes during epididymal maturation and reproducible sperm quality tests suitable for microscope software analysis.

\section{Acknowledgments}

With special affection and appreciation for E.B.-O. (R.I.P.). Without his guidance, friendship, and comments, the elaboration of this work would not have been possible.

This work was financed by Comisión Nacional de Investigación Científica y Tecnológica (CONICYT) Scholarship RHB and Financial Support for Doctoral Thesis Folio 21120861. Fondef CONICYT-grant D08I1076.

\section{Supplementary Data}

Supplementary data related to this article can be found at http://dx.doi.org/10.1016/j.jevs.2015.10.002.

\section{References}

[1] Perreault SD, Wolff RA, Zirkin BR. The role of disulfide bond reduction during mammalian sperm nuclear decondensation in vivo. Dev Biol 1984;101:160-7.

[2] Bustos-Obregón E, Leiva S. Chromatin packing in normal and teratozoospermic human ejaculated spermatozoa. Andrologia 1983; $15: 468-78$.

[3] Mahi C, Yanagimachi R. Induction of nuclear decondensation of mammalian spermatozoa in vitro. J Reprod Fertil 1975;44:293-6.

[4] Barrera C, Mazzolli AB, Pelling C, Stockert JC. Metachromatic staining of human sperm nuclei after reduction of disulphide bonds. Acta Histochem 1993;94:141-9.

[5] Fornés MW, Bustos-Obregón E. Study of nuclear decondensation of the rat spermatozoa by reducing agents during epididymal transit. Andrologia 1994;26:87-92.

[6] Rodriguez H, Bustos Obregón E. Seasonality and freezability vs routine parameters in stallion semen. Histol Histopathol 1986;11:427-30.

[7] Dias GM, Retamal CA, Tobella L, Arnholdt ACV, López ML. Nuclear status of immature and mature stallion spermatozoa. Theriogenology 2006;66:354-65.

[8] Bench GS, Friz AM, Corzett MH, Morse DH, Balhorn R. DNA and total protamine masses in individual sperm from fertile mammalian subjects. Cytometry 1996;23:263-71.

[9] Pirhonen A, Linnala-Kankkunen A, Mäenpää PH. Comparison of partial amino acid sequences of two protamine 2 variants from stallion sperm structural evidence that the variants are products of different genes. FEBS Lett 1989;244:199-202.

[10] Corzett M, Mazrimas J, Balhorn R. Protamine 1: protamine 2 stoichiometry in the sperm of eutherian mammals. Mol Reprod Dev 2002;61:519-27.

[11] Rousseaux J, Rousseaux-Prevost R. Molecular localization of free thiols in human sperm chromatin. Biol Reprod 1995;52:1066-72.

[12] Cleland WW. Dithiothreitol, a new protective reagent for SH groups. Biochemistry 1963;3:480-2.

[13] Kuisma P, Andersson M, Koskinen E, Katila T. Fertility of frozenthawed stallion semen cannot be predicted by the currently used laboratory methods. Acta Vet Scand 2006;48:1-8. 